\title{
Assessment of dabigatran plasma concentration may improve the safety of anticoagulation in patients treated with ibrutinib with concomitant atrial fibrillation
}

\author{
Ewa Lewicka', Paulina Daniluk1', Zofia Lasocka', Ewa Zarzycka², \\ Alicja Dąbrowska-Kugacka', Izabela Nabiałek-Trojanowska'1, Jan M. Zaucha² \\ 1 Department of Cardiology and Electrotherapy, Medical University of Gdańsk, Gdańsk, Poland \\ 2 Department of Hematology and Transplantology, Medical University of Gdańsk, Gdańsk, Poland
}

Correspondence to: Prof. Ewa Lewicka, MD, PhD, Department of Cardiology and Electrotherapy, Medical University of Gdańsk, ul. Dębinki 7, 80-952 Gdańsk, Poland phone: +48583493910, email: ewa.lewicka@gumed.edu.pl Received: March 2, 2020. Revision accepted: March 20, 2020. Published online: March 24, 2020 Kardiol Pol. 2020; 78 (5): 454-457 doi:10.33963/KP.15255 Copyright by the Author(s), 2020
Introduction Ibrutinib is an irreversible Bruton tyrosine kinase inhibitor, which is approved for the treatment of chronic lymphocytic leukemia (CLL), Waldenström macroglobulinemia, and mantle cell lymphoma and is associated with significant improvement in patient outcomes. ${ }^{1}$ However, ibrutinib is related to risk of atrial fibrillation (AF) ranging from $4 \%$ to $16 \%{ }^{2,3} \mathrm{Man}-$ agement of $\mathrm{AF}$ in patients receiving ibrutinib is challenging due to numerous drug interactions. Moreover, ibrutinib inhibits platelet aggregation and increases the risk of bleeding, even without concomitant use of aspirin or anticoagulants, ${ }^{4}$ which makes the decision about stroke prevention more complex. ${ }^{5,6}$ Direct oral anticoagulants (DOACs) have been shown to be at least noninferior to warfarin in the prevention of systemic embolism in patients with $\mathrm{AF}$ and to carry lower bleeding risk. ${ }^{7}$ In the case of dabigatran, it is possible to monitor its plasma concentration, which may be useful in some clinical situations. Nevertheless, current data regarding combination of DOACs and ibrutinib are insufficient to draw firm conclusions, and each case should be assessed individually. 6,8

Methods A 71-year-old man with CLL since 2009 and hypertension started receiving ibrutinib at a standard dose of $420 \mathrm{mg} / \mathrm{d}$ in April 2018. No history of AF was documented, and hypertension was well-controlled (perindopril $5 \mathrm{mg} / \mathrm{d}$ ). Echocardiography showed normal left ventricular ejection fraction of $65 \%$, mild left atrial enlargement $\left(42 \mathrm{~mm} / 24 \mathrm{~cm}^{2}\right)$, and dilatation of the ascending aorta $(53 \mathrm{~mm})$ confirmed by computed tomography angiography. In July 2019, after 15 months on ibrutinib, he reported palpitations and persistent AF was diagnosed. Ibrutinib was withheld, and bisoprolol $5 \mathrm{mg} / \mathrm{d}$ and dabigatran $150 \mathrm{mg}$ twice daily were administered. In September 2019, electrical cardioversion was successfully performed, ibrutinib was resumed, and dabigatran reduced to $110 \mathrm{mg}$ twice daily. After 2 weeks, dabigatran concentration was measured using a commercially available Innovance diluted thrombin time (dTT) assay (Siemens Healthcare Diagnostics $\mathrm{GmbH}$, Erlangen, Germany). Blood samples were taken 3 hours after dabigatran administration (approximately at its peak concentration) and at 12 hours (trough level). During the 6-month follow-up, sinus rhythm was maintained and no bleeding complications were reported.

The second patient, a 70-year-old man with CLL since 1998, started receiving ibrutinib at a standard dose of $420 \mathrm{mg} / \mathrm{d}$ in March 2018. At that time, the patient had hypertension, heart failure (New York Heart Association class II), permanent AF (since 2009, with no attempts to restore sinus rhythm), chronic renal failure, and hypercholesterolemia. Cardiac assessment before starting ibrutinib showed good control of hypertension (telmisartan $80 \mathrm{mg} / \mathrm{d}$ and torasemide $2.5 \mathrm{mg} / \mathrm{d}$ ) and heart rate (bisoprolol 
TABLE 1 Patients' characteristics and laboratory parameters at the time of plasma dabigatran concentration measurement

\begin{tabular}{|c|c|c|}
\hline Parameter & Patient 1 & Patient 2 \\
\hline Age, y & 71 & 70 \\
\hline RAI stage & 1 & 3 \\
\hline Therapies before ibrutinib & 1 & 3 \\
\hline FISH & del17 & del17 \\
\hline Body mass index, kg/m² & 27 & 27 \\
\hline Hypertension & Yes & Yes \\
\hline Renal failure & No & Yes \\
\hline Smoking status & No & No \\
\hline History of AF & No & Yes (permanent) \\
\hline Time since CLL diagnosis, y & 11 & 21 \\
\hline Ibrutinib and dabigatran treatment duration, mo & 6 & 16 \\
\hline $\mathrm{CHA}_{2} \mathrm{DS}_{2}$-VASC & 2 & 3 \\
\hline HAS-BLED ${ }^{a}$ & $1(2)$ & $3(4)$ \\
\hline \multicolumn{3}{|l|}{ First dabigatran concentration measurement } \\
\hline Hemoglobin, g/dl & 15.1 & 14.6 \\
\hline Platelet count,$\times 10^{9} / \mu \mathrm{l}$ & 124 & 179 \\
\hline Creatinine, mg/dl & 0.93 & 1.66 \\
\hline Glomerular filtration rate, $\mathrm{ml} / \mathrm{min} / 1.73 \mathrm{~m}^{2}$ & 82 & 38 \\
\hline Ibrutinib dose, mg & 280 & 280 \\
\hline Dabigatran dose, mg & 110 twice daily & 110 once daily \\
\hline Trough dabigatran concentration, ng/ml & 64 & 48 \\
\hline Peak dabigatran concentration, $\mathrm{ng} / \mathrm{ml}$ & 130 & 145 \\
\hline \multicolumn{3}{|l|}{ Second dabigatran concentration measurement ${ }^{\mathrm{b}}$} \\
\hline Hemoglobin, g/dl & 14.8 & 14.5 \\
\hline Platelet count, $\times 10^{9} / \mu \mathrm{l}$ & 118 & 184 \\
\hline Creatinine, mg/dl & 0.93 & 1.54 \\
\hline Glomerular filtration rate, $\mathrm{ml} / \mathrm{min} / 1.73 \mathrm{~m}^{2}$ & 82 & 45 \\
\hline Ibrutinib dose, mg & 280 & 140 \\
\hline Dabigatran dose, mg & 110 twice daily & 110 once daily \\
\hline Trough dabigatran concentration, ng/ml & 36 & 38 \\
\hline Peak dabigatran concentration, $\mathrm{ng} / \mathrm{ml}$ & 95 & 121 \\
\hline
\end{tabular}

a The score result after including dabigatran treatment

b Two months after the previous measurement

Conversion factors to SI units: to convert creatinine to $\mu \mathrm{mol} / \mathrm{l}$, multiply by 88.4 .

Abbreviations: $\mathrm{AF}$, atrial fibrillation; $\mathrm{CHA}_{2} \mathrm{DS}_{2}$-VASc, congestive heart failure, hypertension, age $\geq 75$ years, diabetes mellitus, history of stroke or thromboembolism, vascular disease, age 65 to 74 years, female sex; CLL, chronic lymphocytic leukemia; FISH, fluorescence in situ hybridization; HAS-BLED, uncontrolled hypertension, abnormal liver or renal function, history of stroke or thromboembolism, history of bleeding, age $\geq 65$ years, use of nonsteroidal anti-inflammatory drugs, and alcohol abuse

$2.5 \mathrm{mg} / \mathrm{d})$. Echocardiography revealed reduced left ventricular ejection fraction of $43 \%$ and significant left atrial enlargement $\left(55 \mathrm{~mm} / 32 \mathrm{~cm}^{2}\right)$. As under previous chemotherapy (chlorambucil, then fludarabine, cyclophosphamide, and rituximab), the patient developed bruising and minor bleedings. No anticoagulation was prescribed until October 2018, when dabigatran was administered at a dose of $110 \mathrm{mg}$ twice daily. As mild bruising occurred, dabigatran was reduced 
to $110 \mathrm{mg} / \mathrm{d}$. After 2 weeks, dabigatran concentration was assessed at its peak and trough levels: 3 and 23 hours after dabigatran administration, respectively. During the 16 -month follow-up, no new bleeding complications were reported.

The study on ibrutinib use in AF was approved by the local ethics committee, and all study participants provided written informed consent.

Results and discussion Clinical characteristics of the study patients are presented in TABLE 1 , which also shows laboratory parameters at the time when plasma dabigatran concentration was measured.

Ibrutinib has a favorable toxicity profile, but increased severity of arrhythmia, AF in particular, and bleeding risk due to its use have been reported. ${ }^{4,9}$ In a retrospective study, which included 582 patients treated with ibrutinib for hematologic malignancies, 76 individuals developed $A F$, and the estimated cumulative incidence of AF at 6 months, 1 year, and 2 years was $5.9 \%, 7.5 \%$, and $10.3 \%$, respectively. There were 9 major bleedings in 7 patients and 34 clinically relevant nonmajor bleedings in 24 patients. $^{3}$

In patients with nonvalvular $\mathrm{AF}$, oral anticoagulation is limited to DOACs and warfarin, but DOACs are preferred over warfarin due to their safety and noninferiority in stroke prevention. ${ }^{7}$ Warfarin is metabolized primarily by the hepatic CYP2C9 enzyme, thus ibrutinib should have no pharmacokinetic interactions, as opposed to DOACs. The anticoagulant effect of warfarin has the advantage of easy reversal, but warfarin is associated with a higher risk of intracranial bleeding compared with DOACs. ${ }^{9}$

Ibrutinib is metabolized by the hepatic CYP3A4 cytochrome, which also applies to rivaroxaban and apixaban. Competition for CYP3A4 between ibrutinib and these DOACs, leading to unpredictable plasma levels of 1 agent or another, may increase the bleeding risk. ${ }^{9}$

Ibrutinib is a P-glycoprotein inhibitor and may increase serum DOAC concentrations, which can be clinically significant in the presence of renal failure, particularly for dabigatran due to its mainly renal (80\%) elimination.

Nevertheless, among the available DOACs, dabigatran appears to be the most suitable drug, as it does not interact with the cytochrome P450 system, has an effective antidote, and its plasma levels can be monitored. ${ }^{7,10}$ Idarucizumab is a specific reversal agent for dabigatran in patients who develop life-threatening bleeding. ${ }^{\text {? }}$ Regarding coagulation tests, at therapeutic plasma levels, dabigatran prolongs the activated partial thromboplastin time (aPTT), and also has a slight effect on the prothrombin time. ${ }^{11}$ Of note, basic coagulation tests, such as aPTT and prothrombin time, should not be performed to assess the anticoagulant effects of DOACs. These tests are not specific for DOACs, and the results depend on the type of the reagent used for testing. The aPTT gives only quantitative information about the presence of dabigatran and may be nonprolonged if a reagent used for its assessment is not sensitive enough. ${ }^{12}$ The dTT assay is the best qualitative method to evaluate the anticoagulant effects of dabigatran and, based on that, calculate dabigatran plasma concentration. In both our patients treated with ibrutinib, we evaluated dabigatran concentration at its trough and peak levels and it was within the therapeutic range of values indicated by the laboratory. Nevertheless, a reference therapeutic range for dabigatran to mitigate the risk of both thromboembolic events and bleeding has not been determined yet. However, it has been suggested that a trough dTT longer than 65 s (corresponding to the dabigatran concentration of $200 \mathrm{ng} / \mathrm{ml}$ ) is associated with a higher bleeding risk. ${ }^{13}$ No stroke nor bleeding events were reported during the mean 11.5-month follow-up, and remission of CLL was observed in both patients. Thus, follow-up examinations demonstrated that the drug concentration was effective and safe during the ibrutinib therapy, also in the patient with renal impairment. Because the patients' clinical condition was stable, and their treatment remained unchanged, no further measurements of dabigatran concentration were performed.

In this study, we showed that the use of dabigatran at its controlled plasma concentration helps prevent stroke in patients with AF treated with ibrutinib. This approach allows clinicians to evaluate the anticoagulant activity and may improve the safety of anticoagulation.

\section{ARTICLE INFORMATION}

\section{CONFLICT OF INTEREST None declared.}

OPEN ACCESS This is an Open Access article distributed under the terms of the Creative Commons Attribution-NonCommercial-NoDerivatives 4.0 International License (CC BY-NC-ND 4.0), allowing third parties to download articles and share them with others, provided the original work is properly cited, not changed in any way, distributed under the same license, and used for noncommercial purposes only. For commercial use, please contact the journal office at kardiologiapolska@ptkardio.pl.

HOW TO CITE Lewicka E, Daniluk P, Lasocka Z, et al. Assessment of dabigatran plasma concentration may improve the safety of anticoagulation in patients treated with ibrutinib with concomitant atrial fibrillation. Kardiol Pol. 2020; 78: 454457. doi:10.33963/KP.15255

\section{REFERENCES}

1 Burger JA, Tedeschi A, Barr PM, et al. Ibrutinib as initial therapy for patients with chronic lymphocytic leukemia. N Engl J Med. 2015; 373: 2425-2437.

2 Ganatra S, Sharma A, Shah S, et al. Ibrutinib-associated atrial fibrillation. JACC Clin Electrophysiol. 2018; 4: 1491-1500.

3 Wiczer TE, Levine LB, Brumbaugh J, et al. Cumulative incidence, risk factors, and management of atrial fibrillation in patients receiving ibrutinib. Blood Adv. 2017; 1: 1739-1748

4 Shatzel IJ, Olson SR, Tao DL, et al. Ibrutinib-associated bleeding: pathogenesis, management and risk reduction strategies. J Thromb Haemost. 2017; 15: 835-847.

5 Chai KL, Rowan G, Seymour JF, et al. Practical recommendations for the choice of anticoagulants in the management of patients with atrial fibrillation on ibrutinib. Leuk Lymphoma. 2017; 58: 2811-2814.

6 Thorp BC, Badoux X. Atrial fibrillation as a complication of ibrutinib therapy: clinical features and challenges of management. Leuk Lymphoma. 2018; 59: 311-320. 
7 Undas A, Drabik L, Potpara T. Bleeding in anticoagulated patients with atrial fibrillation: practical considerations. Pol Arch Intern Med. 2020; 130: 47-58.

8 Visentin A, Campello E, Scomazzon E, et al. Dabigatran in ibrutinib-treated patients with atrial fibrillation and lymphoproliferative diseases: Experience of 4 cases. Hematol Oncol. 2018; 36: 801-803.

9 Boriani G, Corradini P, Cuneo A, et al. Practical management of ibrutinib in the real life: Focus on atrial fibrillation and bleeding. Hematol Oncol. 2018; 36: 624-632.

10 Quintavalla R, Lombardi M, Prandoni P, et al. Increased dabigatran plasma concentration during ibrutinib treatment: a case of cerebral hemorrhage and successful dabigatran reversal by idarucizumab. Aging Clin Exp Res. 2018; 30: 93-95.

11 Douketis J, Wang G, Chan N, et al. Effect of standardized perioperative dabigatran interruption on the residual anticoagulation effect at the time of surgery or procedure. J Thromb Haemost. 2016; 14: 89-97.

12 Tripodi A, Braham S, Scimeca B, et al. How and when to measure anticoagulant effects of direct oral anticoagulants? Practical issues. Pol Arch Intern Med. 2018; 128: 379-385.

13 Huisman MV, Lip GY, Diener HC, et al. Dabigatran etexilate for stroke prevention in patients with atrial fibrillation: resolving uncertainties in routine practice. Thromb Haemost. 2012; 107: 838-847. 